\title{
A biologically inspired controller
}

\author{
James P. Carneal and Chris R. Fuller \\ Vibration and Acoustics Laboratories, Mechanical Engineering Department, Virginia Polytechnic Institute \\ and State University, Blacksburg, Virginia 24061-0238
}

(Received 24 May 1994; revised 18 October 1994; accepted 5 January 1995)

\begin{abstract}
A biologically inspired control approach for reducing vibrations in distributed elastic systems has been derived and experimentally verified for narrow-band excitation. The control paradigm approximates natural biological systems for initiating movement, in that a low number of signals are sent from an advanced, centralized controller (analogous to the motor cortex of the brain) and are then distributed by local simple rules to multiple control actuators (analogous to muscle fiber). Both theoretical and experimental investigations of three different local rules were carried out including a stability analysis for reducing beam vibrations. In general the results have demonstrated that the biological control approach has the potential to control multimodal response in distributed elastic systems using an array of many actuators with a reduced order main controller. Thus significant reductions in control system computational complexity have been realized by this approach. (C) 1995 Acoustical Society of America.
\end{abstract}

PACS numbers: $43.40 . \mathrm{Vn}$

\section{INTRODUCTION}

Recent work has demonstrated the potential of active control of distributed elastic systems using multiple, independent actuators and sensors. In work concerned with the control of sound radiation from vibrating panels, the importance of the number of control channels and optimization of the transducer position and shape has been demonstrated. ${ }^{1}$ However, these investigations were carried out for a fixed frequency and it is apparent that for good control over a bandwidth of frequencies, the control actuators and sensors need to be adaptive in shape. At first sight this problem could be solved using an overall transducer broken up into many individual small elements each connected by an individual control channel. In this situation the control transducer would effectively reoptimize its configuration for different conditions by adaptively weighting each transducer segment. Meirovitch and Norris ${ }^{2}$ have demonstrated the advantage of such an approach by considering fully distributed control in reducing control spillover. The disadvantage of this approach is that, for systems with a high modal density, the number of actuators and sensors required becomes extremely large. A high number of control channels has a number of problems mainly associated with memory requirements and computational time in the hardware systems used to implement the control. In addition, collinearity of transducer transfer functions causes stability problems in systems with a high number of transducers.

A new approach of controlling distributed elastic systems is presented. The approach is inspired by the hierarchy of biological natural systems when initiating movement. A simplified model can be stated as follows: ${ }^{3}$ A movement signal originates in a single motor neuron in the motor cortex of the brain. This signal is transmitted along an axon to diverging neuronal circuits in the spinal cord which thereby excites multiple anterior motor neurons. The signal from each of the anterior motor neurons stimulates more diverging neuronal circuits which are connected to multiple muscle fibers. In concept, a signal for movement is generated in the motor cortex of the brain and is distributed by external diverging neuronal circuits to multiple muscle fibers.

This paper details a biologically inspired (BIO) control approach, where a centralized processor generates a control signal (analogous to the motor cortex of the brain) which is distributed by local simple rules (analogous to diverging neuronal circuits) to multiple control actuators (analogous to muscle fibers). The plant to be controlled is a distributed elastic system that is harmonically excited and controlled by multiple control inputs. As seen in Fig. 1, the BIO control approach has one control input chosen as the "master" which is under direction of the centralized controller. The other "slave" inputs derive their control laws by localized, simple rules related to the behavior of their neighbor actuators and are proportional to the centralized controller direct signal. Note that this approach has been previously studied in a limited analytical investigation ${ }^{4}$ and experimentally verified. $^{5}$

This type of control system is related to a hierarchical control approach, which is summarized as follows: The main objective of the hierarchical control system is shared by all levels. Decentralized decision making units exist at all levels due to the complexity of the main control objective, and limited information handling capabilities of each unit. Also, several of these units can operate in parallel. ${ }^{6}$ One can see the similarities in a hierarchical control system and a biologically inspired control system. However, there is one significant difference. In a hierarchical control approach, the time horizon of interest increases as one goes up the hierarchy, i.e., the lower levels of the hierarchy are concerned with short-term goals whereas the top level of the hierarchy is concerned with long-term goals. Conversely, the time horizon of interest for the biologically inspired control system is the same for all levels (the centralized control processor and the local learning rules), while the complexity of the controller changes at each level. Therefore to distinguish the ap- 


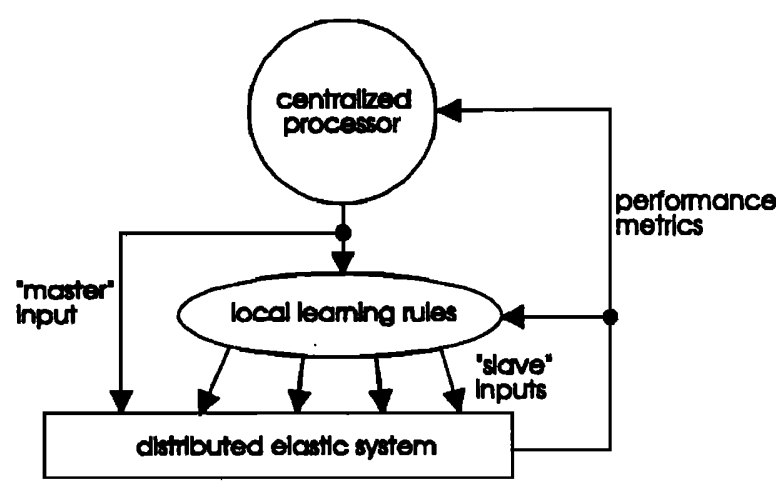

FIG. 1. Biological control approach.

proach from formal hierarchical control the approach was named "a biologically inspired" controller. 4

In this paper, the theory is developed for the master control input and two local control approaches, the "phase variation" and "optimal distribution" methods, followed by a stability analysis. The control approach is then analytically applied to a beam, where a third local control method is developed, the "moment distribution" method. An experimental investigation to control beam vibration is presented including a comparison between theoretical and experimental control performance. Concluding remarks are then presented.

\section{THEORY}

The control system theory is developed for the biologically inspired controller, with an emphasis on the effects of driving multiple actuators with one main control channel. To gain insight on multiple actuator-single-channel control system performance, a frequency domain LMS algorithm was used to derive the control theory since it incorporates the control path transfer functions into the update equation. First, the master control input is analyzed, followed by the addition of the phase variation method. Since the optimal and moment distribution methods have the same structure, only the optimal distribution will be analyzed.

\section{A. Master control input}

The control system analysis for the master actuator is a frequency domain analysis for a single output adaptive feedforward LMS control system, following Widrow and Stearns ${ }^{7}$ and Boucher et al. ${ }^{8}$ As seen in Fig. 2, the complex error (e) can be described as the sum of the primary disturbance (d) and contributions from the control sources:

$$
\mathbf{e}\left(\omega_{0}\right)=\mathbf{d}\left(\omega_{0}\right)+\mathbf{H}\left(\omega_{0}\right) \mathbf{w}\left(\omega_{0}\right),
$$

where $\mathbf{H}$ denotes the control loop transfer function, $\mathbf{w}$ is the output of the complex adaptive filter, and $\omega_{0}$ is the excitation frequency. The system is assumed to be steady state and the disturbance is assumed to be single frequency harmonic; therefore the dependence of the variables on $\omega_{0}$ will be dropped for convenience. error,

By defining the cost function $(J)$ as the square of the

$$
J=\mathbf{e}^{h} \mathbf{e}=\mathbf{d}^{h} \mathbf{d}+\mathbf{d}^{h} \mathbf{H} \mathbf{w}+\mathbf{w}^{h} \mathbf{H}^{h} \mathbf{d}+\mathbf{w}^{h} \mathbf{H}^{h} \mathbf{H w}
$$

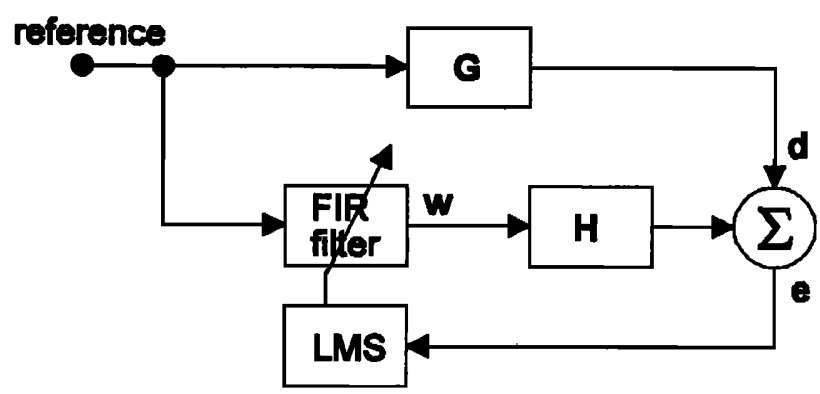

FIG. 2. Traditional adaptive feedforward control system schematic.

(where the superscript $h$ denotes the Hermitian transpose), a quadratic function of the adaptive filter $(w)$ is formed with a unique global minimum. Taking the derivative of the cost function $(J)$ with respect to the adaptive filter $(\mathbf{w})$,

$$
\frac{\partial J}{\partial \mathbf{w}}=2 \mathbf{H}^{h} \mathbf{d}+2 \mathbf{H}^{h} \mathbf{H} \mathbf{w}=2 \mathbf{H}^{h} \mathbf{e},
$$

the optimal adaptive filter can be found by

$$
\mathbf{w}_{\text {opt }}=-\frac{\mathbf{H}^{h} \mathbf{d}}{\mathbf{H}^{h} \mathbf{H}} \text {. }
$$

From the standard gradient descent technique, the update equation for the adaptive filter at iteration $k$ is written as

$$
\mathbf{w}_{k+1}=\mathbf{w}_{k}+\mu\left(-\nabla_{k}\right)
$$

where $\mu$ is the convergence coefficient and $\nabla_{k}$ is the gradient of the mean-square error performance surface. However, the gradient can be defined as

$$
\dot{\nabla}_{k}=\frac{\partial J}{\partial \mathbf{w}}=2 \mathbf{H}^{h} \mathbf{e}
$$

Therefore the update equation becomes

$$
\mathbf{w}_{k+1}=\mathbf{w}_{k}-2 \mu \mathbf{H}^{h} \mathbf{e} \text {. }
$$

In traditional MIMO adaptive feedforward control, the adaptive filter would be represented by the complex vector $\mathbf{w}$, having a number of elements equal to the number of control channels (degrees of freedom). In theory, the number of error sensors (e) would have to be greater than or equal to the number of control sources, and the control loop transfer function (H) would be a matrix.

In the biologically inspired controller, the adaptive filter (w) for the master control channel remains a single output complex quantity, with the additional degrees of freedom implemented downstream of the update equation through the use of local control approaches. Note that we have not discussed a procedure for choosing the particular actuator to which the master signal is to be applied. This could be achieved, for example, by a simple search scheme.

\section{B. Local control approaches}

Once the master control channel is updated, the control inputs to the slave channels are derived using local rules. Two local rules are first analyzed, the phase variation and the optimal distribution methods. The phase variation method varies the phase of the master control input, while 


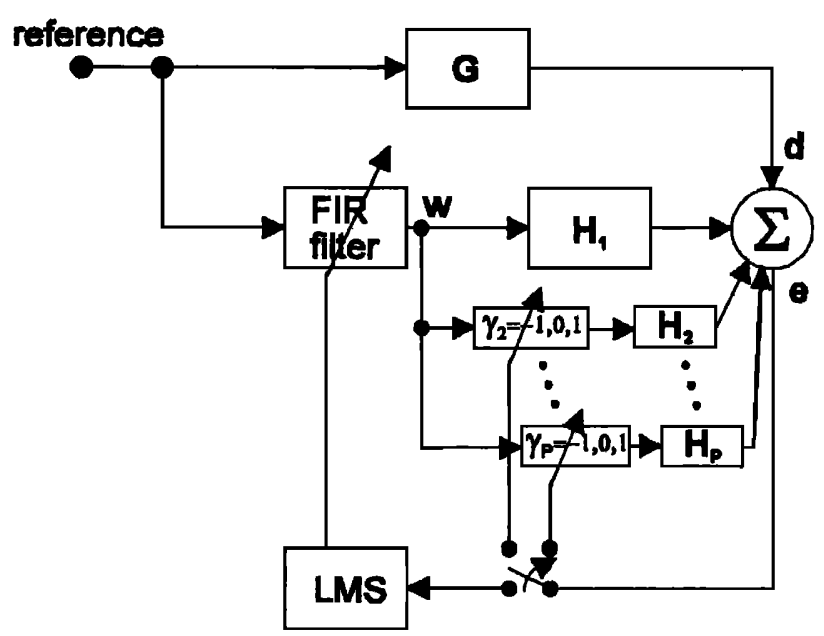

FIG. 3. Phase variation control system schematic.

holding the magnitude constant, and applies this control input to the slave control paths. The optimal distribution allows both the magnitude and phase of the master control input to be varied in application to the slave control paths, and is derived offline from linear quadratic optimal control theory.

Mathematically, the local control approach can be expressed as complex gains $(\gamma)$ applied to the master control channel to determine the control inputs applied to the slave control paths. Note that the method of determining these gains is not limited to the above methods, but could also include experimental, neural network, and genetic algorithm methods.

\section{Phase variation}

The following approach for a local rule is based on the observation that, for a distributed elastic system with little damping, the phase between two arbitrary points on the structure will be close to 0 (in phase) or $\pi$ (out of phase). Thus the local approach is to take the optimal master control input, vary the phase, and apply it to a slave control path. Figure 3 shows the schematic for the phase variation control system. Using the traditional adaptive feedforward control system schematic (seen in Fig. 2) as a baseline, note that $\mathbf{H}_{1}$ is now defined as the master control path. Also, note the addition of the slave control paths $\mathbf{H}_{2}$ to $\mathbf{H}_{P}$, along with gains $\gamma_{2}$ to $\gamma_{P}$, where $P$ is the total number of control paths.

The phase variation algorithm is defined as follows. With the master control input locked at its optimal value, the slave control input is varied in phase $(\gamma=+1)$, out of phase $(\gamma=-1)$ or turned off $(\gamma=0)$ while the cost function $J$ is observed for each change. The condition that results in the lowest cost function is kept and the process is then applied to the next slave control path until all slave control paths are progressively tested. Note that the sequence of activating the slave paths is not defined, and the control performance depends on this sequence accordingly. By this method, a distributed actuator with a generalized function that drives a response similar to the uncontrolled vibration distribution with low control spillover is constructed. Note that the pre-

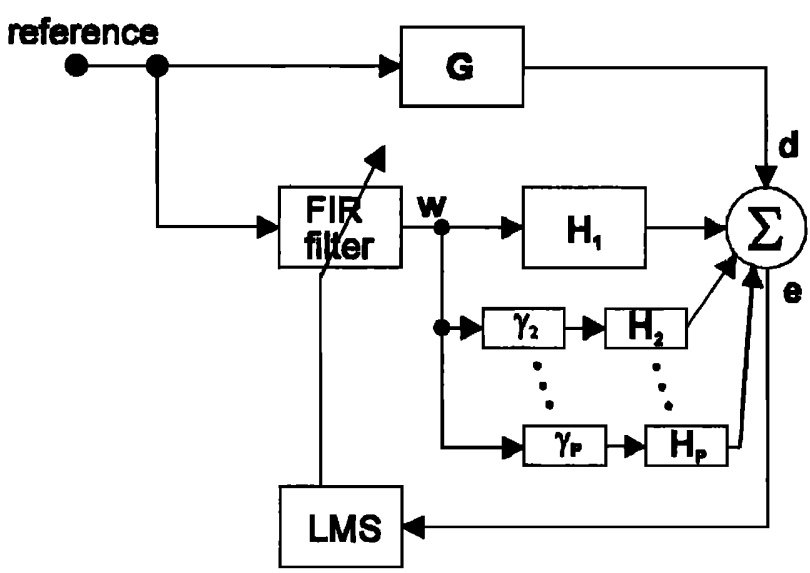

FIG. 4. Optimal distribution control system schematic.

vious process is suboptimal as are many biological systems. However, it is believed that good control performance will still be obtained.

Since the master control channel is allowed to converge to an optimal value before the application of the phase variation algorithm, the update for the adaptive filter remains the same as the single output case,

$$
\mathbf{w}_{k+1}=\mathbf{w}_{k}-2 \mu \mathbf{H}_{1}^{h} \mathbf{e},
$$

where $\mathbf{H}_{1}$ denotes the master control channel transfer function.

\section{Optimal distribution}

The optimal distribution local rule was solved a priori and was developed from MIMO linear quadratic optimal control theory. Essentially the measured or calculated control path transfer functions between the actuators and sensors are used to calculate the theoretical optimal gains. These gains are then scaled relative to the master channel. The weights of the adapative filter of the master channel can then be adapted to minimize the cost function and simultaneously the slave control signals will be derived by proportion. Written in terms of gains applied to the slave actuators $(\gamma)$ and the master control channel adaptive filter (w), this forms a system of linear equations:

$$
\left\{\begin{array}{c}
-\mathbf{H}_{1}^{h} \mathbf{d} \\
\vdots \\
-\mathbf{H}_{P}^{h} \mathbf{d}
\end{array}\right\}=\left[\begin{array}{ccc}
\mathbf{H}_{1}^{h} \mathbf{H}_{1} & \cdots & \mathbf{H}_{1}^{h} \mathbf{H}_{P} \\
\vdots & \ddots & \vdots \\
\mathbf{H}_{P}^{h} \mathbf{H}_{1} & \cdots & \mathbf{H}_{P}^{h} \mathbf{H}_{P}
\end{array}\right]\left\{\begin{array}{c}
\gamma_{1} \\
\vdots \\
\gamma_{P}
\end{array}\right\} \mathbf{w},
$$

where $\mathbf{d}$ and $\mathbf{H}_{i}$ are defined in the preceding section. The solution to this linear system of equations provides a leastsquares solution of the optimal gains. The gains are then scaled so that the gain of the master control channel $\left(\gamma_{1}\right)$ is 1 .

The implementation of the optimal distribution into the control system is shown in Fig. 4. The control signal fed to the master control input (w) is operated on by the optimal complex gains $\left(\gamma_{2} . . \gamma_{P}\right)$ and fed to the slave transfer functions $\left(\mathbf{H}_{2} . . \mathbf{H}_{P}\right)$. With the addition of the slave control paths, the error can now be written 


$$
\mathbf{e}_{k}=\mathbf{d}_{k}+\left(\mathbf{H}_{1}+\sum_{i=2}^{P} \gamma_{i} \mathbf{H}_{i}\right) \mathbf{w}_{k},
$$

therefore leading to a new update equation for the adaptive filter:

$$
\mathbf{w}_{k+1}=\mathbf{w}_{k}-2 \mu\left(\mathbf{H}_{1}+\sum_{i=2}^{P} \gamma_{i} \mathbf{H}_{i}\right)^{h} \mathbf{e} .
$$

It is evident from the above equation that the update equation for the master control input is now dependent upon the additional slave control paths.

\section{Stability analysis}

\section{Master control input}

Following the frequency domain analysis of Widrow and Stearns ${ }^{7}$ and Boucher et al. ${ }^{8}$ the coordinates of the error surface can be translated into principle coordinates defined by the eigenvalues of the system by expanding the expected value of the matrix $H^{h} \mathbf{H}$ into $\mathbf{Q} \Lambda \mathbf{Q}^{h}$, where $\mathbf{Q}$ is a matrix of eigenvectors and $\Lambda$ is a diagonal matrix of eigenvalues. The rotated and translated set of coordinates can be defined by

$$
\mathbf{v}=\mathbf{Q}^{H}\left(\mathbf{w}-\mathbf{w}_{\text {opt }}\right) \text {. }
$$

The update equation can now be written in terms of the principle coordinates of the error surface:

$$
\mathbf{v}_{k}=[\mathbf{I}-\mathbf{2} \mu \Lambda]^{k} \mathbf{v}_{0},
$$

whereby definition the eigenvalue matrix $(\Lambda)$ is diagonal. This leads to an independent set of converging eigenvalues so that

$$
\nu_{m, k}=\left(1-2 \mu \lambda_{m}\right)^{k} \nu_{m, 0},
$$

where $\nu_{m}$ is the coordinate of the $m$ th eigenvector corresponding to the $m$ th eigenvalue $\left(\lambda_{m}\right)$. For stability, the update of $\nu_{m}$ should be bounded, or

$$
\lim _{k \rightarrow \infty}\left(1-2 \mu \lambda_{m}\right)^{k}=0 \quad \forall m,
$$

leading to the inequality

$$
\left|1-2 \mu \lambda_{m}\right|<1 \quad \forall m
$$

or

$$
0<\mu<1 / \lambda_{m} \quad \forall m .
$$

Therefore upper bound of the convergence parameter $(\mu)$ is limited by the maximum eigenvalue $\left(\lambda_{\max }\right)$.

\section{Phase variation}

As detailed above, the phase variation method is invoked after the master control input has converged to an optimal value. While the master control input is converging, stability bounds are determined by the above analysis for the master control adaptive filter (w) which is continuous and has no bound. Since the adaptive gains $(\gamma)$ and slave control inputs have no influence on the master control input, the convergence parameter $(\mu)$ is limited by the maximum eigenvalue $\left(\lambda_{\max }\right)$, which is derived from the master control input transfer function $\left(\mathbf{H}_{1}\right)$.
Once the phase variation method is invoked, the adaptive filter (w) is locked at its optimal value. The phase variation method then applies the optimal filter from the master channel to the slave channels operated on by a discrete and bounded gain $(\gamma)$ which must have the value $-1,0$, or +1 . By definition, bounded gains must be stable; therefore the phase variation method is stable.

\section{Optimal distribution}

For the optimal distribution method, the above analysis for the update equation shows that the matrix $\mathbf{H}^{h} \mathbf{H}$ must include the slave channel gains $(\gamma)$ and transfer functions $\left(\mathbf{H}_{i}\right)$, written as

$$
\mathbf{H}^{h} \mathbf{H}=\left(\mathbf{H}_{1}+\sum_{i=2}^{P} \gamma_{i} \mathbf{H}_{i}\right)^{h}\left(\mathbf{H}_{1}+\sum_{i=2}^{P} \gamma_{i} \mathbf{H}_{i}\right) .
$$

This matrix can be into expanded into $\mathbf{Q} \Lambda \mathbf{Q}^{h}$, similar to the above analysis, and likewise, the upper bound of the convergence parameter $(\mu)$ is limited by the maximum eigenvalue $\left(\lambda_{\max }\right)$.

By definition, the matrix $\mathbf{Q} \Lambda \mathbf{Q}^{h}$ is symmetric and has a orthonormal set of eigenvectors. Also, the matrix must have positive eigenvalues since it is a physical system. From these facts, it is evident that the addition of other control inputs will increase the largest eigenvalue in the matrix, therefore decreasing the upper bound on the convergence parameter $(\mu)$. It is apparent that the determination of the convergence parameter for the optimal distribution is case specific and depends on the values of the adaptive gains $(\gamma)$ and the master and slave control paths $\left(\mathbf{H}_{1}\right.$ through $\left.\mathbf{H}_{P}\right)$.

\section{ANALYTICAL APPLICATION TO VIBRATION CONTROL}

The biologically inspired control theory is specialized to a specific case of a 1-D distributed elastic system (a beam) with simply supported boundary conditions. The disturbance is provided by a point force that excites the system harmonically and narrow band, where the control inputs are provided by piezoelectric actuators. Certain specifics relevant to this application are presented including a new local rule (moment distribution) and details required for the stability analysis. For a more complete derivation, refer to Fuller and Carneal. ${ }^{4}$

\section{A. Local control approach}

\section{Optimal distribution}

Upon application of the optimal distribution to control of total beam out-of-plane vibrational energy density, an interesting phenomenon became apparent. As can be seen in Fig. 5, the optimal distribution approximates the static moment distribution in a simply supported beam when a point force is applied. At first it was thought that the optimal distribution would follow the modal response of the beam at the excitation frequency, dominated by certain modes as determined by the excitation frequency. However, a thorough investigation of Eq. (9) has revealed that the distribution is dependent on the contributions of all the modes, even when the system is excited on or very near resonance. The impor- 


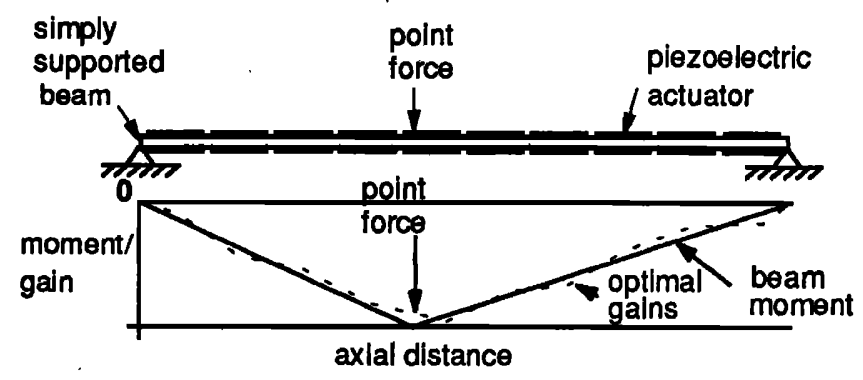

FIG. 5. Beam optimal gain distribution.

tant observation was that the optimal distribution is independent of frequency. This phenomenon is discussed in the following paragraph.

In the Introduction, the advantages of fully distributed control were discussed. Another way to look at this control mechanism is as follows: A harmonic disturbance sets up a disturbance field (i.e., a displacement or moment distribution) in the structure. To achieve total control, the control actuators must exactly recreate that disturbance field, but out of phase. Two examples are given. Example 1 is where the disturbance and the control actuator are both point forces and colocated on the structure. By definition of colocation, the disturbance and the control actuator set up the same field in the structure (whether that is a displacement, shear, or moment field). The control amplitude is the disturbance amplitude and the control phase is 180 deg relative to the disturbance phase. The optimal adaptive filter [defined in Eq. (4)] is -1 and therefore the control input is independent of frequency. Example 2 is where the disturbance and control actuators impart different fields into the structure and are not colocated. The disturbance is a point force and the control actuator imparts a fully distributed moment in the structure. Since the control actuator is fully distributed over the structure, it is able to fully recreate the disturbance moment field and therefore the disturbance displacement field (the displacement field is the second integral of the moment field). As in example 1, the control amplitude is proportional to the disturbance amplitude and the control phase is 180 relative to the disturbance phase and therefore the control input is independent of frequency. The important concept is that the control field must emulate the disturbance field, whether it is a displacement, moment, or acoustic field. How close the control field is to the disturbance field determines the maximum attainable amount of control. For an arbitrary disturbance, a fully distributed actuator will achieve the best control. ${ }^{2}$

Due to the above discussion, it is evident that for the optimal and moment distributions to be independent of frequency, the control actuators must approximate a fully distributed actuator. Also, the disturbance must be fixed spatially and it should also be noted that the distributions are disturbance dependent.

\section{Moment distribution}

As a simplification of the above local rule, the moment distribution method was devised and based on the internal beam moment, which is zero at the boundary and linear to

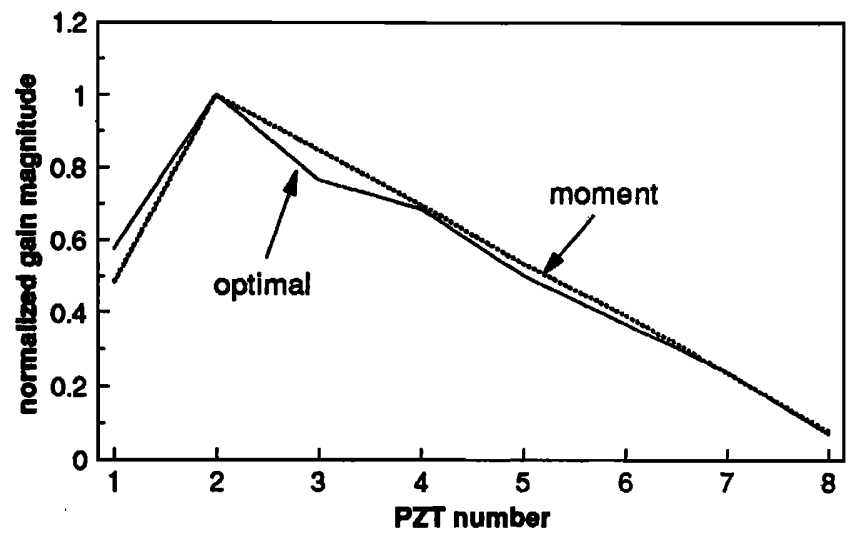

FIG. 6. Comparison of beam optimal and moment gains.

the position of the point force. The contribution of the $i$ th actuator $\left(\gamma_{i}\right)$ was derived from its position relative to the point force, defined as

$$
\begin{aligned}
& \gamma_{i}=\frac{x_{p, i}}{x_{d}}, \quad \forall x_{p}<x_{d}, \\
& \gamma_{i}=\frac{x_{p, i}-l}{x_{d}-l}, \quad \forall x_{p}>x_{d},
\end{aligned}
$$

where $x_{p}$ is the position of the control input (in this case the center of the PZT), $x_{d}$ is the position of the disturbance, and $l$ is the length of the beam. The gains were then scaled so that the gain of the master control channel $\left(\gamma_{1}\right)$ was 1 . As with the optimal distribution, this distribution was independent of frequency (note that the aforementioned conditions apply). However, unlike the optimal distribution, the moment distribution was easily calculable since its form is simply a gain of constant slope. A comparison of optimal and moment distributions as calculated from Eqs. (9) and (19), respectively, is presented in Fig. 6. Note the slight variations in the moment distribution as compared to the optimal distribution. These discrepancies are due to the finite number of actuators used in the calculation of the optimal distribution. It should be noted that as the number of actuators increases, the optimal distribution will approach the moment distribution.

One disadvantage of the optimal and moment distributions are that the distributions are determined a priori, which results in a decrease in the adaptability of the controller (a change in the disturbance spatially will render the distribution nonoptimal). However, this local control approach provides the best possible attenuation for a specific number of control channels and can be applied wherever there is a spatially fixed disturbance and the main controller adapts primarily to changes in frequency.

\section{B. Stability}

It was previously determined that the maximum convergence parameter for stability would be less for the BIO controller using the optimal distribution than for a single output control system. It was also noticed that the determination of the convergence parameter was case specific and depends on the values of the complex adaptive gains $(\gamma)$ and the master and slave control paths $\left(\mathbf{H}_{1}\right.$ through $\left.\mathbf{H}_{P}\right)$. To verify this theo- 


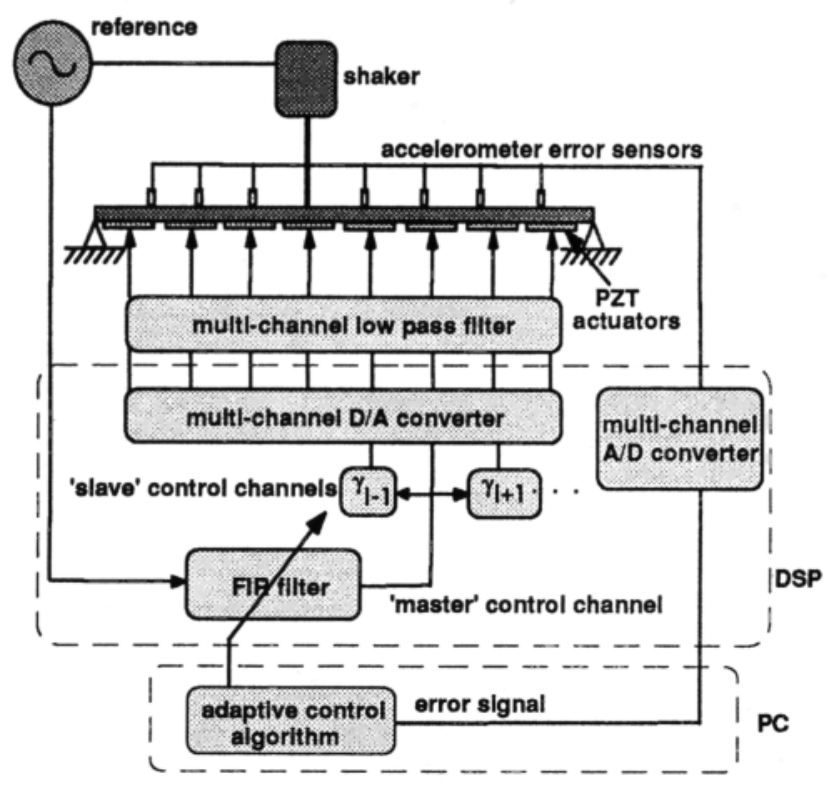

FIG. 7. Experimental schematic of biologically inspired controller.

retical development, the maximum convergence parameter was calculated for the BIO controller optimal distribution and a single output system. The adaptive gains were calculated as per Eq. (9) with the master and slave transfer functions calculated as in Fuller and Carneal. ${ }^{4}$ However, the master and slave transfer functions do not include several other transfer functions (i.e., accelerometer calibration, amplifier gain and phase, etc.) that are critical in matching the experimental maximum convergence parameter $\left(\mu_{\max }\right)$. It was determined that to correctly predict the experimental $\mu_{\max }$, all of the transfer functions from the control voltage generated at the D/A converters on the digital signal processor (DSP), seen in Fig. 7, to the accelerometer voltage input to the A/D converters on the DSP had to be taken into account. These transfer functions were measured independently and incorporated into the theoretical model. Experimental versus theoretical comparisons of the maximum convergence parameter for stability are presented later.

\section{EXPERIMENTAL INVESTIGATION}

\section{A. Experimental setup}

The implementation of the biologically inspired (BIO) controller is detailed, followed by a description of the experimental apparatus for the beam vibration control experiments.

\section{Controller}

The BIO controller was programmed on a TMS320C30 digital signal processor (DSP) mounted in a $8038633-\mathrm{MHz}$ personal computer (PC). To increase the maximum sampling rate, the number of operations performed by the DSP was minimized. Therefore the I/O functions, the cost function, and FIR filter calculations were performed on the DSP, while the controller logic was performed on the PC and communicated to the DSP. The sample rate for the controller was set at approximately $10 \times$ the excitation frequency with each error signal consisting of 250 time sample points, leading to an update for the FIR filter approximately every $0.05 \mathrm{~s}$ at a sampling frequency of $5000 \mathrm{~Hz}$. Figure 7 shows the experimental schematic of the BIO controller for the vibration control experiment.

As stated earlier, Sec. I used a frequency domain analysis to include the control path transfer function $(\mathbf{H})$ to determine the effect of the additional control paths on the update equation. However, due to the computational inefficiency of a frequency domain controller, a time-averaged gradient (TAG) LMS algorithm was chosen for the physical implementation of the biologically inspired control algorithm. The update equation used for the TAG LMS control algorithm was defined in Eq. (5) with the gradient $(\nabla)$ at iteration $k$ being defined as the finite difference approximation of the change in time-averaged cost function $(J)$ with respect to the vector of FIR filter coefficients $(w)$, written as

$$
\nabla_{k}=\frac{\Delta J}{\Delta \mathbf{w}}=\left(\frac{J_{k}-J_{k-1}}{\mathbf{w}_{k}-\mathbf{w}_{k-1}}\right) .
$$

The time-averaged cost function is defined as

$$
J_{k}=\frac{1}{N} \sum_{t=1}^{N} J_{t},
$$

where $J_{t}$ is the cost function at time step $t$, and $N$ is the total number of time sample points. Note that the use of the TAG LMS algorithm does not directly include the control path transfer function $(\mathbf{H})$ in the gradient or the adaptive filter update equation. However, it can be shown that the finite difference approximation of the gradient in Eq. (20) is equivalent to the gradient defined in Eq. (6) as $\Delta \mathbf{w} \rightarrow 0$. Therefore when applied to a physical system, the frequency domain controller and the TAG LMS controller will converge to the same optimal adaptive filter $\left(\mathbf{w}_{\text {opt }}\right)$ and stability bounds are comparable.

Again referring to Fig. 7, the BIO control algorithm is discussed. For the "master channel only" case, the adaptive gains $(\gamma)$ were set to zero, rendering the control algorithm as a single output system. For the phase variation method, the master channel was allowed to converge, then the FIR filter was locked at its optimum value. The master channel FIR filter coefficients were applied to the slave control channel, one by one, alternating left then right of the master control channel (this progression was earlier determined to provide the best performance). The filter coefficients were tried in phase $(\gamma=+1)$, out of phase $(\gamma=-1)$, or turned off $(\gamma=0)$ while the cost function $(J)$ was observed for each change. The gain $(\gamma)$ that resulted in the lowest cost function was kept, the cost function was measured, and the process was applied to the next slave control channel until all slave control paths were progressively tested.

TABLE I. Beam properties.

\begin{tabular}{cc}
\hline \hline & Properties \\
\hline Material & steel \\
Dimensions (m) & $0.380 \times 0.038 \times 0.0046$ \\
Density $\left(\mathrm{kg} / \mathrm{m}^{3}\right)$ & 7860 \\
Damping & 0.02 \\
Elastic modulus (GPa) & 200 \\
\hline \hline
\end{tabular}




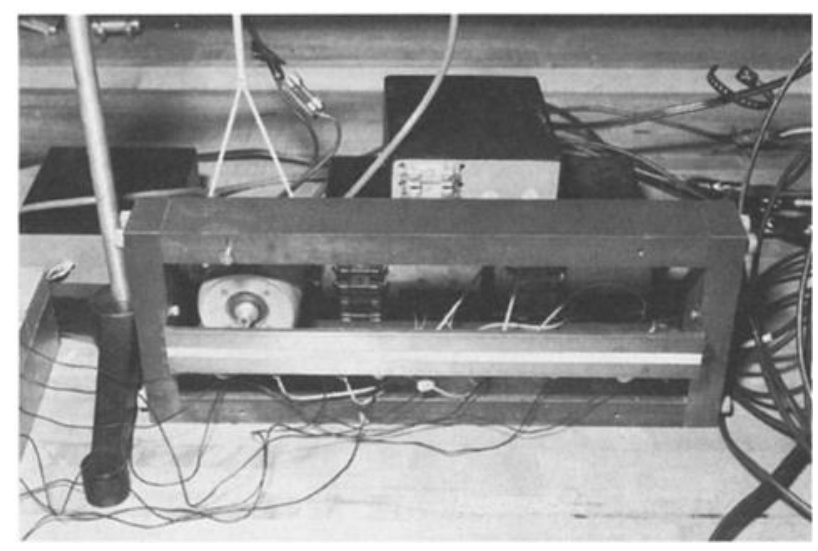

FIG. 8. Photograph of beam experimental setup.

For the optimal and moment distributions, the adaptive gains $(\gamma)$ were determined a priori and then scaled relative to the master actuator. The gains were entered to the control program prior its execution. Once the FIR filter update equation was invoked, the master channel FIR filter was updated as described in Eqs. (5), (20), and (21). However, before the digital-to-analog conversion process was performed, the master channel FIR filter coefficients were multiplied by the adaptive gains $(\gamma)$, thereby generating the slave control outputs. When the D/A process was triggered, the master and slave control signals were output simultaneously.

\section{Vibration control}

To experimentally verify the BIO controller applied to vibration control, an experiment was performed on a steel beam of length $0.380 \mathrm{~m}$, width $0.038 \mathrm{~m}$, and thickness $0.0048 \mathrm{~m}$, the properties of which are seen in Table I. A top front view photograph of the experimental setup is shown in Fig. 8, which shows the test beam mounted on a rig with shims (which allowed rotation of the boundary, but little transverse displacement) in order to approximate simply supported boundary conditions. Harmonic, narrow-band excitation was provided by an approximation of a point force through a shaker mounted at $0.051 \mathrm{~m}$. Experiments were performed at a variety of on- and off-resonance excitation frequencies; however, for brevity, detailed results are only presented for the excitation frequencies of 400 and $604 \mathrm{~Hz}$. As seen in Table II, both of these test frequencies are off resonance between the second and third beam modes.

As seen in Fig. 9, error signals were provided by the output of seven matched accelerometers located along one side of the beam at evenly spaced locations. To approximate a cost function of beam vibrational energy density, a sum of the mean-square voltage read from the accelerometers was

TABLE II. Beam natural frequencies.

\begin{tabular}{ccc}
\hline \hline Mode & $\begin{array}{c}\text { Theoretical } f_{n} \\
(\mathrm{~Hz})\end{array}$ & $\begin{array}{c}\text { Experimental } f_{n} \\
(\mathrm{~Hz})\end{array}$ \\
\hline 1 & 73.6 & 76 \\
2 & 288.3 & 292 \\
3 & 646.1 & 644 \\
4 & 1146.4 & 1130 \\
\hline \hline
\end{tabular}

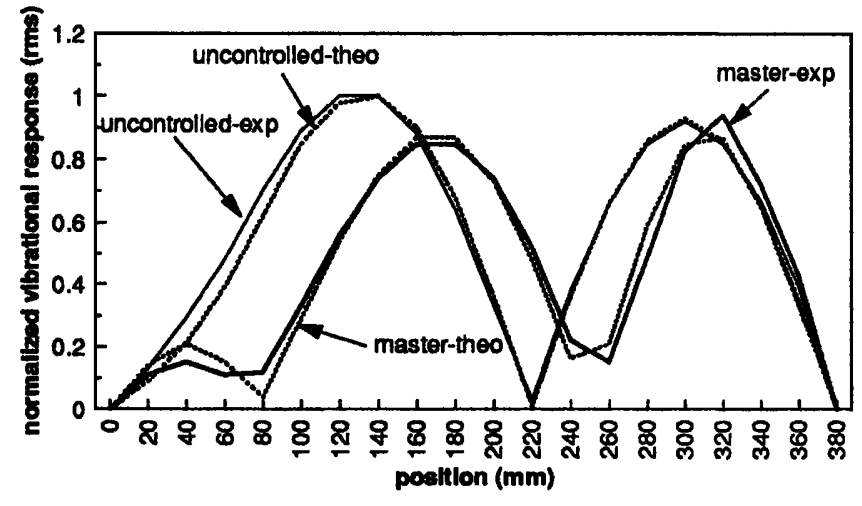

FIG. 9. Experimental and theoretical normalized beam vibrational response for uncontrolled and master tests.

provided to the LMS algorithm (and the phase variation method of the BIO controller). The cost function at time step $t$ is defined as

$$
J_{t}=\sum_{i=1}^{M}\left|\nu_{i}\right|^{2}
$$

where $\nu_{i}$ is the accelerometer voltage, the subscript $i$ indicates the accelerometer number index, and $M$ is the total number of accelerometers.

Control inputs were provided by eight ceramic piezoelectric (PZT) patches each having dimensions $0.032 \mathrm{~m}$ long $\times 0.038 \mathrm{~m}$ wide $\times 2.59 E-04 \mathrm{~m}$ thick, the properties of which are provided in Table III. The PZT patches were directly bonded to the beam at evenly spaced central locations. Previous experiments have determined that the one-sided PZT actuators were as effective as the two-sided actuators for exciting transverse motion in a beam. ${ }^{9}$ Therefore to avoid mass loading of the beam, the PZT actuators were bonded to one side of the beam only, instead of the usual PZT antisymmetric pair.

\section{B. Performance metrics}

The BIO controller performance was analyzed in terms of reduction in cost function, elastic system transverse vibration, and convergence parameter for a stable system. For a benchmark comparison, controller performance was compared to the performance of a conventional MIMO filtered- $X$ LMS controller.

\section{Controller performance}

Performance of the control algorithm was defined as the reduction in the cost function as

TABLE III. PZT properties.

\begin{tabular}{cc}
\hline & Properties \\
\hline Material & lead zirconate titanate \\
Density $\left(\mathrm{kg} / \mathrm{m}^{3}\right)$ & 7600 \\
Strain coefficient $(\mathrm{m} / \mathrm{V})$ & $166 E-12$ \\
Elastic modulus $\left(\mathrm{N} / \mathrm{m}^{2}\right)$ & $63 E+9$ \\
\hline \hline
\end{tabular}




$$
\text { reduction }(\mathrm{dB})=10 \log _{10}\left(\frac{J_{\text {uncontrolled }}}{J_{\text {controlled }}}\right) \text {, }
$$

where $J_{\text {uncontrolled }}$ is the uncontrolled cost function and $J_{\text {controlled }}$ is the controlled cost function.

For a more detailed analysis of the response of the elastic system, a laser vibrometer was used to determine the transverse vibration for the uncontrolled and controlled cases. For the vibration control test, measurements were taken at 21 evenly spaced central axial points on the beam described above. A modal decomposition ${ }^{10}$ of the beam vibrational data was performed to gain further insight on controller behavior.

\section{Controller convergence}

In the above analysis section, it was predicted that the maximum convergence parameter $\left(\mu_{\max }\right)$ for a stable single output system would be greater than the maximum convergence parameter $\left(\mu_{\max }\right)$ for a stable BIO controller using the optimal (or moment) distribution. Experiments were performed to verify this trend.

To determine the maximum convergence parameter, the control program was run with the convergence parameter set at different values. The program was allowed to continue until it was determined to be unstable, marginally stable, or stable. The maximum convergence parameter was defined as the largest value that resulted in a stable system.

To determine the stability of the system, the variation in cost function was determined, which was defined as difference in the maximum and minimum cost function over several iterations of the update equation [Eq. (5)]. The stability of the system was divided into three categories: unstable, marginally stable, and stable. A stable algorithm was defined as the variation in cost function being less than $5 \%$ of the uncontrolled cost function. A marginally stable algorithm was defined as the variation in cost function being greater than 5\% of the uncontrolled cost function, but the maximum cost function did not increase with time. This is consistent with the "characteristic oscillatory behavior" defined in Elliot $e$ a $a .^{11}$ An unstable system was defined as the maximum cost function increasing with time.

\section{Experimental procedure}

Several different test cases were performed including off-resonance and on-resonance frequency excitation for the master control channel only case and all of the local rules. However, the experimental procedure for a set of tests was the same and is detailed by performance metric (i.e., controller performance and controller convergence).

\section{Controller performance}

The experimental procedure for the master and phase variation test case was as follows: First, the disturbance was turned on. The control program was initialized: the convergence parameter $(\mu)$ was set at approximately 0.01 , the master control actuator was chosen, and the sampling frequency was set at approximately $10 \times$ the excitation frequency. The uncontrolled elastic system out-of-plane vibrational velocity was then measured using the laser vibrometer, and the uncontrolled cost function was measured. After the invocation of the control algorithm, the master control channel was allowed to converge; then the cost function and the elastic system vibration were measured for the master case. The phase variation algorithm was invoked, and after completion, the cost function and the elastic system vibration was measured for the phase variation case. The controller was then turned off.

For the other local rules (the optimal and moment distributions), the disturbance was set at approximately the same level. The control program were initialized: the convergence parameter $(\mu)$ was set at approximately 0.01 , the master control channel was set to the desired value, and the sampling frequency was set at $10 \times$ the excitation frequency. The uncontrolled elastic system vibration and cost function were then measured. After the controller was invoked and allowed to converge, the elastic system vibration and cost function were measured for the controlled case.

\section{Controller convergence}

The experimental procedure for the controller convergence test was as follows. The program was initialized: the step size, the master actuator, and the sampling frequency were set to desired values. The uncontrolled cost function was recorded; then the control algorithm was invoked. While recording the cost functions at each iteration, the algorithm was allowed to continue until it was determined to be unstable, marginally stable, or stable.

\section{RESULTS}

An extensive investigation was performed for the BIO controller applied to vibration control; however, for brevity, only select results are presented. It should be noted that the results presented are indicative of the overall trends observed, and in general, the stated conclusions apply to all the results attained.

\section{A. Selection of master actuator}

Analytical models were run to assess the influence of the selection of the master actuator on the phase variation performance. Analytical cases were run at a frequency of 400 $\mathrm{Hz}$ with each PZT actuator chosen as the master actuator for use in the phase variation algorithm. Table IV shows the performance of the master actuator, the increase in performance due to the phase variation method, and the control string. The control string of the phase variation method denotes the final implementation of the slave control channels, where 0 indicates off, $\oplus$ indicates the master control channel (which is by definition in phase), + indicates channels implemented in phase, and - indicates channels implemented out of phase.

As can be seen in Table IV, the final control performance varied greatly with the choice of master actuator from a low of $0.0 \mathrm{~dB}$ for actuators 4 and 8 to a high of $9.8 \mathrm{~dB}$ for actuator 2. It is evident that the good performance of actuator 2 was a result of the PZT's colocation with the disturbance force. However, this unique situation of colocated distur- 
TABLE IV. Influence of master actuator on phase variation performance $(400 \mathrm{~Hz})$.

\begin{tabular}{cccc}
\hline \hline $\begin{array}{c}\text { Master } \\
\text { actuator }\end{array}$ & $\begin{array}{c}\text { Master test } \\
\text { performance }(\mathrm{dB})\end{array}$ & $\begin{array}{c}\text { Phase variation } \\
\text { increase }(\mathrm{dB})\end{array}$ & Control string $^{\mathrm{a}}$ \\
\hline 1 & 6.3 & 0 & $\oplus 0000000$ \\
2 & 9.8 & 0.2 & $0 \oplus 0+0000$ \\
3 & 8.0 & 2.7 & $+0 \oplus 00000$ \\
4 & 0.0 & 2.5 & $---\oplus++++$ \\
5 & 1.9 & 1.2 & $0000 \oplus+00$ \\
6 & 5.0 & 2.7 & $-00+0 \oplus 0+$ \\
7 & 1.6 & 6.0 & $-00+++0 \oplus-$ \\
8 & 0.0 & 6.8 & $---++++\oplus$ \\
\hline \hline
\end{tabular}

Note: $\oplus$ indicates the master control channel.

bance and actuator is not representative of real control situations, nor is it a true test of distributed control performance.

The increase in control performance achieved with the addition of the phase variation method varied from a low of 0 to a high of $6.8 \mathrm{~dB}$, seen in Table IV. Note that for several choices of master actuator, the phase variation method performance increase was less than $3 \mathrm{~dB}$. This was attributed to the limited degrees of freedom associated with fixing the magnitude and varying the phase, as dictated by the phase variation method.

Using the results of Table IV, actuator 7 was chosen to be the master actuator since that actuator best exhibited the capabilities of the phase variation method and had marginal control performance by itself.

\section{B. Controller performance}

In this section, the relative control performance for control with the master actuator only (referred to as master) with the local rules of the phase variation, the optimal distribution, and the moment distribution methods are compared. For these experiments, the excitation frequency was $400 \mathrm{~Hz}$, the master channel was actuator 7 , and the sampling frequency was $4000 \mathrm{~Hz}$. Theoretical and experimental controller performance seen in Table II, Table V, Fig. 9, and Fig. 10 show the same trends and similar magnitudes; therefore only the experimental results will be discussed.

As seen in Table V, the master case, where there was only one control input, shows poor control performance of $1.5 \mathrm{~dB}$. Beam vibrational response of the master case, shown in Fig. 9, exhibits control spillover in the beam axial location of 160-240 mm. The modal decomposition of beam vibrational response, seen in Fig. 11, shows a significant reduction in mode 2 with significant spillover into mode 3 . It is evident that the master actuator (PZT 7) can effectively couple into

TABLE V. Biologically inspired controller performance $(400 \mathrm{~Hz})$.

\begin{tabular}{ccc}
\hline \hline Method & Theoretical $(\mathrm{dB})$ & Experimental $(\mathrm{dB})$ \\
\hline Master (\#7) & 1.6 & 1.5 \\
Phase variation & 7.6 & 6.6 \\
Control string & $-00+++\oplus-^{\mathrm{a}}$ & $-00+++\oplus-^{\mathrm{a}}$ \\
Optimal & 36.2 & 24.8 \\
Moment & 27.5 & 23.6 \\
\hline \hline
\end{tabular}

${ }^{a}$ Note: $\oplus$ indicates the master control channel.

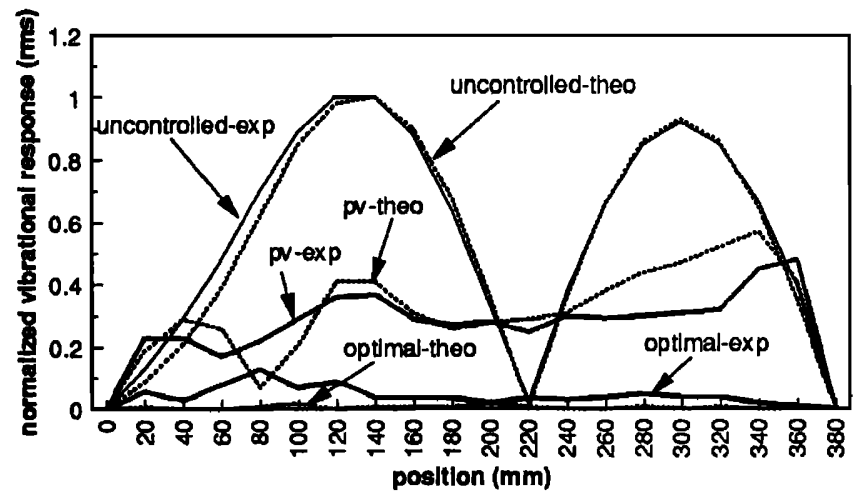

FIG. 10. Experimental and theoretical normalized beam vibrational response for uncontrolled, phase variation, and optimal tests.

mode 2 due to its location along the beam near an antinode of the second mode. However, this location was also at an antinode of the third mode, and therefore some of the control energy was used to increase the response of this mode.

While maintaining the control system simplicity of one control channel, the BIO controller using the phase variation method, shows admirable control performance of an additional $5.1 \mathrm{~dB}$ of attenuation as seen in Table $\mathrm{V}$. In addition to the master actuator (PZT 7) implemented in phase, there were three slave control channels implemented out of phase and three slave control channels implemented in phase, as seen in the control string. Beam vibrational response of the phase variation case, seen in Fig. 10, shows little control spillover with significant reductions in beam response. The modal decomposition of beam vibrational response, seen in Fig. 11, shows reductions in modes 2 and 3 , with a slight increase in mode 1 response. It is apparent that the addition of the slave control actuators were able to couple into mode 3 , thereby increasing control performance compared to the master case. From these results, it is evident that the phase variation method constructed a distributed actuator (by varying the phase of the master actuator and holding magnitude constant) that coupled effectively into the uncontrolled modes of the beam.

The optimal distribution shows good control performance of $24.8 \mathrm{~dB}$, as seen in Table $\mathrm{V}$ with little or no in-

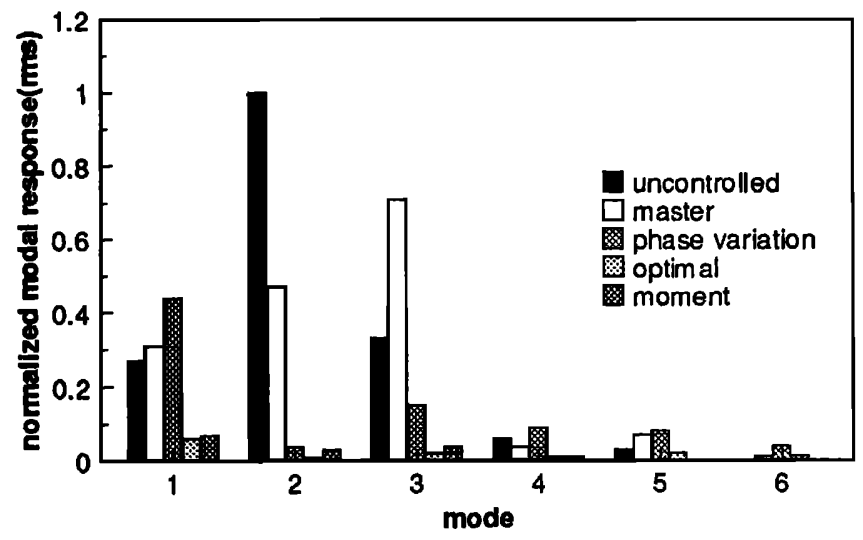

FIG. 11. Modal response of beam for uncontrolled, master, phase variation, optimal and moment tests. 
crease in control system complexity. Beam vibrational response of the optimal distribution method, seen in Fig. 10, shows a significant reduction in beam vibrational response with no control spillover. The modal decomposition of beam vibrational response, displayed in Fig. 11, shows total reductions in all excited modes. It is obvious that the distributed actuator formed by the optimal distribution method was able to couple effectively into all uncontrolled modes of the beam with no control spillover.

The moment distribution, which was an approximation of the optimal distribution, exhibited slightly poorer control performance than the optimal distribution, as expected. As seen in Table $\mathrm{V}$, control performance was still quite good at $23.6 \mathrm{~dB}$. The modal decomposition of beam vibrational response, seen in Fig. 11, shows the same general reduction in modes as the optimal distribution with total reductions in all excited modes. Although the distributed actuator formed by the moment distribution was able to effectively couple into all the uncontrolled modes of the beam, the slight differences due to the approximation (of the optimal distribution) resulted in slightly less reduction of the excited modes.

It was stated earlier that the distributed actuator formed by the optimal and moment distributions was independent of frequency. Tests performed over a wide range of test frequencies and configurations indicated that the optimal distribution provided approximately $24 \mathrm{~dB}$ of attenuation for offresonance harmonic excitation. The moment distribution provided slightly less attenuation of approximately $23 \mathrm{~dB}$. It is evident from the exemplary performance over a wide range of test parameters that the optimal and moment distributions are frequency independent. Note that the disturbance must be fixed spatially and the distribution is disturbance dependent.

\section{Controller convergence and stability}

In the previous analysis, it was determined that the maximum convergence parameter $\left(\mu_{\max }\right)$ for a stable single output system would be greater than the maximum convergence parameter $\left(\mu_{\max }\right)$ for a stable BIO controller using the optimal (or moment) distribution. This is due to the additional control inputs of the optimal (or moment) distribution. Experiments were run to determine the maximum convergence parameter that could be used for a stable system for the master case and the optimal distribution. For these tests, the system was excited at $604 \mathrm{~Hz}$, the master channel was actuator 7, and the sampling period was $6000 \mathrm{~Hz}$.

Table VI shows the maximum convergence parameter obtained for a single output system (the master case) and the optimal distribution. For both theoretical and experimental methods, the master (single output) control method has a maximum convergence parameter $\left(\mu_{\max }\right)$ one order of magnitude greater than the optimal distribution $\mu_{\max }$, which verifies the trends previously predicted. Note that the theoretically predicted magnitudes are similar to the experimentally determined magnitudes for a marginally stable system rather than a stable system. It is evident that the definition put forth in this paper for a marginally stable system is actually the theoretical determination between a stable and an unstable system as defined in Elliot et al. ${ }^{11}$ They state that as the
TABLE VI. Maximum convergence parameter $(604 \mathrm{~Hz})$.

\begin{tabular}{lccc}
\hline \hline & \multicolumn{3}{c}{ Maximum convergence parameter } \\
\cline { 2 - 4 } $\begin{array}{l}\text { Control } \\
\text { method }\end{array}$ & theoretical & $\begin{array}{c}\text { experimental- } \\
\text { marginally stable }\end{array}$ & $\begin{array}{c}\text { experimental- } \\
\text { stable }\end{array}$ \\
\hline Master & 0.353 & 0.21 & .07 \\
Optimal & 0.039 & 0.03 & .004 \\
\hline \hline
\end{tabular}

convergence parameter approaches the maximum value for stability, the control system exhibits a "characteristic oscillatory behavior," which is consistent with the definition of the marginally stable system in this paper.

\section{CONCLUSIONS}

A biologically inspired control approach for reducing vibrations in distributed elastic systems has been experimentally verified for narrow-band excitation. The control approach, inspired by biological systems, approximated the control structure used with biological muscle, where a few main control signals were sent from an advanced, centralized controller (the brain) and distributed by local rules to multiple actuators (muscle tissue). Experimental investigations of performance of three different variations of local rules were carried out including controller convergence considerations.

For vibration control, comparisons with a theoretical analysis showed good agreement of the performance trends. Performance of the various methods decreased vibration levels in a simply supported beam by up to $25 \mathrm{~dB}$ for offresonance harmonic excitation for a single main channel of control. Also, the distributed actuator formed by the optimal and moment distributions was verified to be independent of frequency over a wide range of test frequencies and configurations. Stability bounds for the controller were shown to be predictable and the predicted trend of decreasing maximum convergence parameter (for stability) with the additional control inputs of the BIO controller was verified.

In general the results demonstrated the biological control approach has the potential to control multimodal response in distributed elastic systems using an array of actuators with a reduced order controller. Thus significant reductions in control system computational complexity have been realized by this approach. Future work will concentrate on additional local rules and theoretical application to radiated sound power control of 2-D distributed elastic systems.

\section{ACKNOWLEDGMENTS}

The authors gratefully acknowledge the support of this work by The Structural Acoustics Branch at NASA Langley Research Center, Dr. Harold Lester, Technical Monitor.

\footnotetext{
${ }^{1}$ R. L. Clark, "Advanced sensing techniques for active structural acoustic control," Ph.D. dissertation, VPI\&SU (1992).

${ }^{2}$ L. Meirovitch and M. A. Norris, "Vibration control," Proceedings of Inter-Noise 84 (Noise Control Foundation, Poughkeepsie, NY, 1984), pp. 477-482.

${ }^{3}$ B. R. Landau. Essential Human Anatomy and Physiology (Scott, Foresman, Glenview, IL, 1976), pp. 191-192.
} 
${ }^{4}$ C. R. Fuller and J. P. Carneal, "A biologically inspired control approach for distributed elastic systems," J. Acoust. Soc. Am. 93, 3511-3513 (1993).

${ }^{5}$ J. P. Carneal and C. R. Fuller, "A biologically inspired controller for sound and vibration applications," presented at the 35th Structures, Structural Dynamics and Materials Conference Adaptive Structures Forum, 1994, AIAA Paper 94-1785 (unpublished).

${ }^{6}$ M. G. Singh, Dynamical Hierarchical Control (North-Holland, Amsterdam, 1980).

${ }^{7}$ B. Widrow and S. Stearns, Adaptive Signal Processing (Prentice-Hall, Englewood Cliffs, NJ, 1985).

${ }^{8}$ C. C. Boucher, S. J. Elliot, and P. A. Nelson, "The Convergence Behavior of a Multichannel Active Noise Control System," Proc. Inst. Acoust. 12, 719-727 (1990).

${ }^{9}$ G. P. Gibbs and C. R. Fuller, "Excitation of thin beams using asymmetric piezoelectric actuators," J. Acoust. Soc. Am. 92, 3221-3227 (1992).

${ }^{10}$ C. H. Hansen, S. D. Snyder, and C. R. Fuller, "Noise reduction of a vibrating square panel by use of active sound sources and active vibration sources," Proceedings of Noise and Vibration 89, Singapore, 1989 (unpublished), pp. E50-E57.

${ }^{11}$ S. J. Elliot, I. M. Strothers, and P. A. Nelson, "A multiple error LMS algorithm and its application to the active control of sound and vibration," IEEE Trans. Acoust. Speech Signal Process. ASSP-35, 1423-1434 (1987). 